\title{
ON SCIURO-HYPNUM OEDIPODIUM AND S. CURTUM
} (BRACHYTHECIACEAE, BRYOPHYTA)

\author{
O SCIURO-HYPNUM OEDIPODIUM И S. CURTUM \\ (BRACHYTHECIACEAE, BRYOPHYTA)
}

\author{
MiChAEL S. IGNATOV $^{1} \&$ IRINA A. MiLYUTINA ${ }^{2}$ \\ МИХАИЛ С. ИГНАТОВ ${ }^{1} \&$ ИРИНА А. МИЛЮТИНА ${ }^{2}$
}

Abstract

\begin{abstract}
Sciuro-hypnum curtum has been synonymized with $S$. oedipodium, but the present revision reveals that they are two distinct species although morphological variation is very broad in both of them. However, molecular delimitation of these two species (nrITS) is much more sharp. Sciurohypnum curtum is a widespread species in boreal forests of Eurasia, and occurs also in North America mainly in its eastern part, with solitary localities in the West. Sciuro-hypnum oedipodium is widespread in western North America, totally absent in the eastern part of this continent, and is known in Russia in Caucasus and in Chukotka. Brachythecium holzingeri is synonymized with $S$. oedipodium.
\end{abstract}

Резюме

Sciuro-hypnum curtum был отнесен с синонимы к S. oedipodium и последние 25 лет не считался самостоятельным видом, но настоящее исследование установило, что эти два вида довольно далеки друг от друга, несмотря на то, что из-за сильной вариабельности некоторые их морфотипы внешне очень сходны. Молекулярные данные по последовательностям ITS подтверждают их весьма значительные отличия, при том что каждый из них константен на протяжении всего ареала. Sciuro-hypnum curtum широко распространен в таежных лесах Евразии, встречается на востоке Северной Америки и в единичных точках на ее западе. Sciuro-hypnum oedipodium широко распространен на западе Северной Америки, отсутствует на востоке этого континента, а также выявлен в России на Кавказе и на Чукотке. Brachythecium holzingeri отнесен в синонимы к S. oedipodium.

\section{INTRODUCTION}

The complex of species around Scuiro-hypnum (formerly Brachythecium) starkei is known to be difficult, causing considerable incongruence of species concepts of different authors during the XIX and most of the XX century.

A detailed overview of this history was presented by Piippo (1983), who also selected the neotype of Hypnum starkei Brid. and lectotypes of $H$. oedipodium Mitt. and $H$. curtum Lindb., i.e. the taxa in which the indefinite concepts were the main source of taxonomic and nomenclatural confusions.

According to Piippo (1983), this complex includes two species in the Northern Hemisphere,
Brachythecium oedipodium and B. starkei (this group of Brachythecium was later transferred to Sciuro-hypnum by Ignatov \& Huttunen (2002)). Both these species are widespread in northern Europe occurring also in Asia and North America. The concept of Piippo received wide acceptance and was followed in checklists of North America (Anderson et al., 1990), China (Redfearn et al., 1996), Europe (Hill et al., 2006), East Europe and North Asia (Ignatov,Afonina, Ignatova et al., 2006).

The work on the treatment of Brachytheciaceae for the Flora of North America, however, revealed certain difficulties in the application of

1 - Main Botanical Garden of Russian Academy of Sciences, Botanicheskaya, 4, Moscow 127276 Russia Россия 27276 Москва, Ботаническая, 4, Главный ботанический сад РАН

2 - A.N.Belozersky' Research Institute of Physico-Chemical Biology, Moscow State University, Moscow 119991

Russia - НИИ Физико-химической биологии им. А.Н. Белозерского, МГУ, Москва 119991 [fax: 7-(095)-

939-31-81; e-mail:tav@genebee.msu.ru] 
Piippo's (1983) concept. The species identical to widespread European 'oedipodium' sensu Piippo was found to be more or less widespread in New England and neighboring areas in eastern North America (but with two localities in the Rocky Mountains), while the type of Hypnum oedipodium was from the mountains of the West. The type itself comprises poorly developed plants, however very numerous collections from the western states, usually kept in North American herbaria (e.g. in DUKE, MO, NY, US) under 'Brachythecium starkei', represent a species quite different from 'oedipodium' sensu Piippo. The type of Hypnum oedipodium, as it will be argued below, belongs to this widespread western species, and therefore cannot be applied to the widespread Eurasian and eastern North American species. The earliest species name for the latter species and also the name used for a long time in Europe is 'curtum', thus this taxon has to be called:

Sciuro-hypnum curtum (Lindb.) Ignatov, comb. nov. - Hypnum curtum Lindb., Musci Scandinavici 35. 1879 [the validation in Helsingfors Dagblad 1878 (342): 2.1878 is earlier, but probably has to be ignored as it appeared in a public newspaper, not in scientific literature] Brachythecium curtum (Lindb.) J. Lange \& C.O.E.Jensen, Medd. Groenland. 3: 337. 1887.

At the same time, Sciuro-hypnum starkei s. str., identical with European and Asian, occurs in North America, although limited in its distribution to the eastern provinces of Canada, occurring in U.S.A. only in Maine, Vermont and Wisconsin.

\section{MORPHOLOGICAL DIFFERENCES BETWEEN}

\section{S. CURTUM AND S. OEDIPODIUM}

Both species are very variable, so the marginal phenotypes and poorly developed plants of these species (as well as many other Brachytheciaceae) are difficult or even impossible to interpret.

The optimally developed, S. curtum (cf. Fig. 1 ) is a relatively large plant with rather remotely arranged leaves, that are patent and usually somewhat twisted along their length when dry, resulting in a peculiar appearance that has an aspect of terrestrial phenotypes of Plagiothecium denticulatum. Branches have an apparent tendency to complanate foliage. Stem leaves are broadly ovate below and are broadest at $1 / 7-1 / 10$ of leaf length. Decurrencies are long but rather narrow. Mid leaf cells are linear, 60-80 $\mu \mathrm{m}$ long, being 7-12:1. Cells in leaf corners are enlarged, thin-walled and thus pellucid, gradually or rather abruptly delimited from laminal and basal juxtacostal cells. Branch leaves are usually strongly serrate. Seta is distinctly and invariably rough. Capsule is relatively long, and when young is strongly curved with operculum pendent to faced back towards the seta, or ultimately even faced upward, the capsule having made a curve of $>270^{\circ}$ [there are however exceptions when capsule is relatively short]. The under-developed plants have about the same size of stem and branches, but smaller and more remote leaves.

The optimally developed plants of $S$. oedipodium (e.g. Fig. 2) are rather densely and terete foliate, so the plants have the appearance of Brachythecium or of a very large Sciuro-hypnum glaciale. Leaves are appressed at their bases, and above are either erect, or gradually reflexed. Leaves are ovate, but compared with $S$. curtum are not that broad and usually have broadest point at $1 / 6-1 / 7$ of leaf length (not at $1 / 7-1 / 10$ or occasionally even below the point of insertion in S. curtum). Decurrencies are long and conspicuously broad (this is a useful character for identification of smaller phenotypes). Mid-leaf cells are very variable even within one shoot, ranging from 3-4:1 to 7-10:1; an example is shown in Fig. 5. Cells in the leaf corner are subquadrate to shortly rectangular, slightly enlarged, rather numerous along the margin to the broadest point of leaf, with 'normally' thickened cell walls, thus the alar group is conspicuous, but 'not especially pellucid, not especially opaque', usually very gradually transforming into laminal and basal juxtacostal cells. Branch leaves usually serrulate, but variable in this character from subentire to quite serrate. Seta is distinctly rough, although in a few collections only slightly so. Capsule is short, inclined to horizontal, and in many cases looks 'too abruptly' hozirontal for that short shape; more rarely the capsule is somewhat elongate, but at the immature state is never strongly curved. The under-developed plants are smaller and with smaller leaves and the variation from large to small shoots is observed in many collections. 


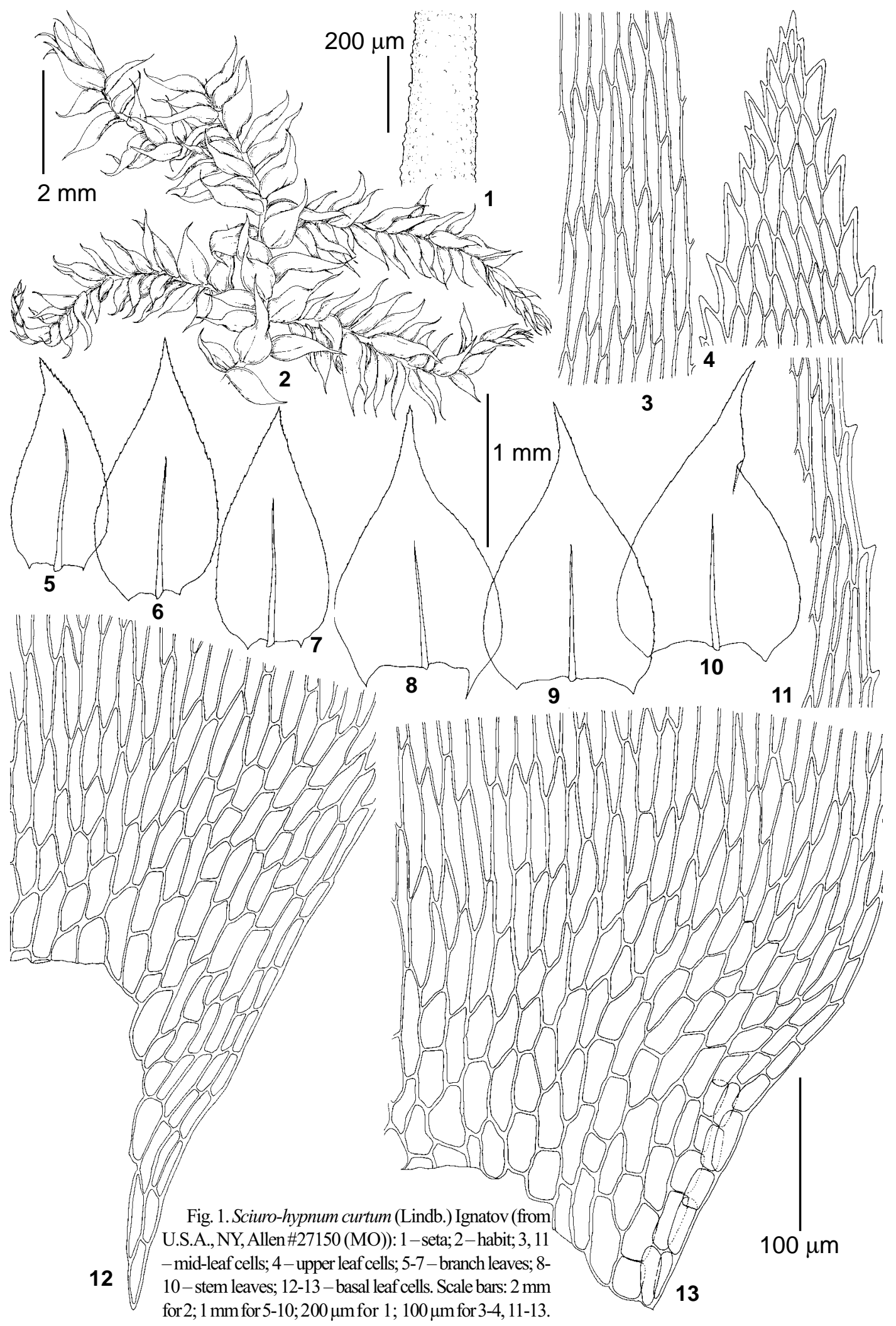




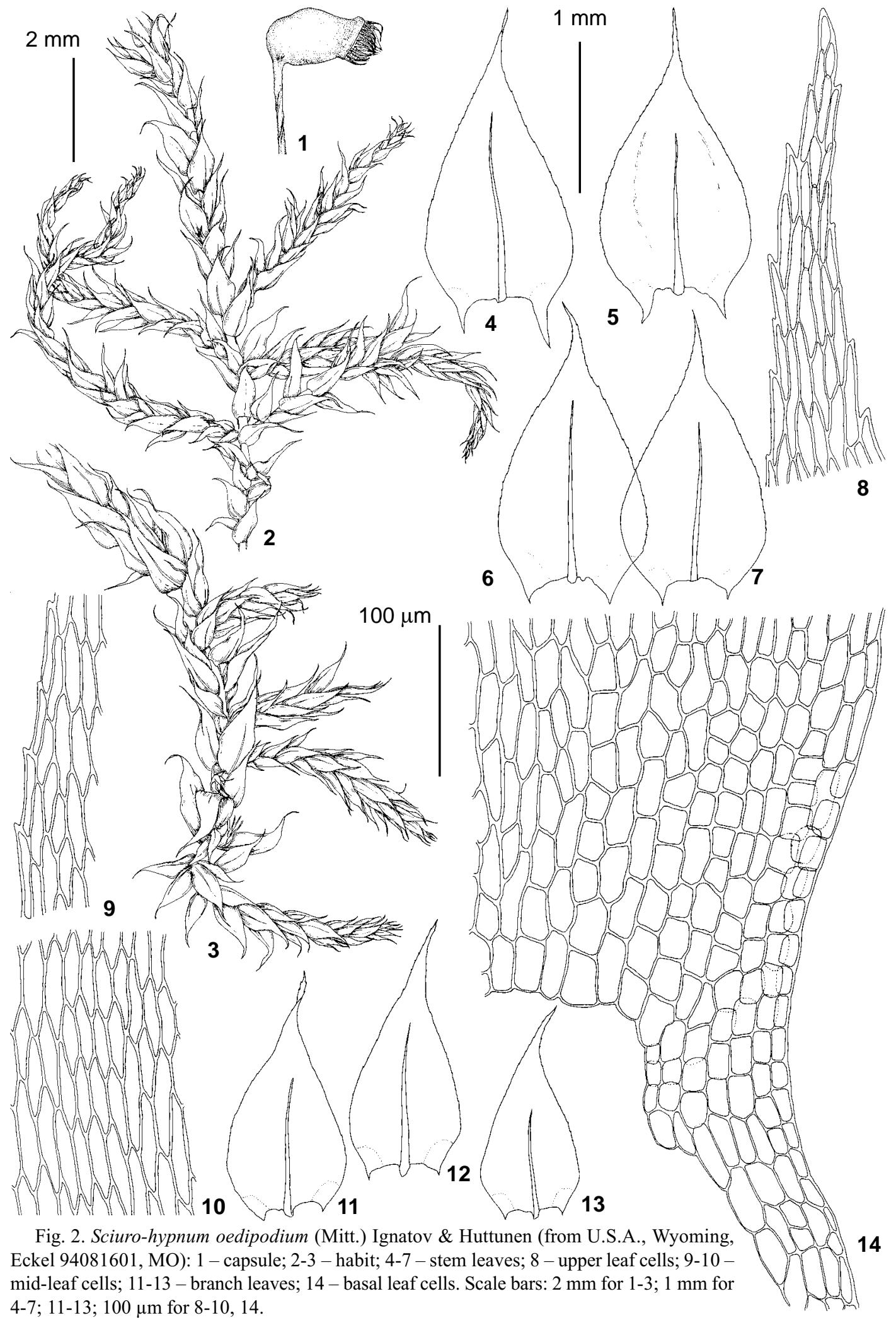




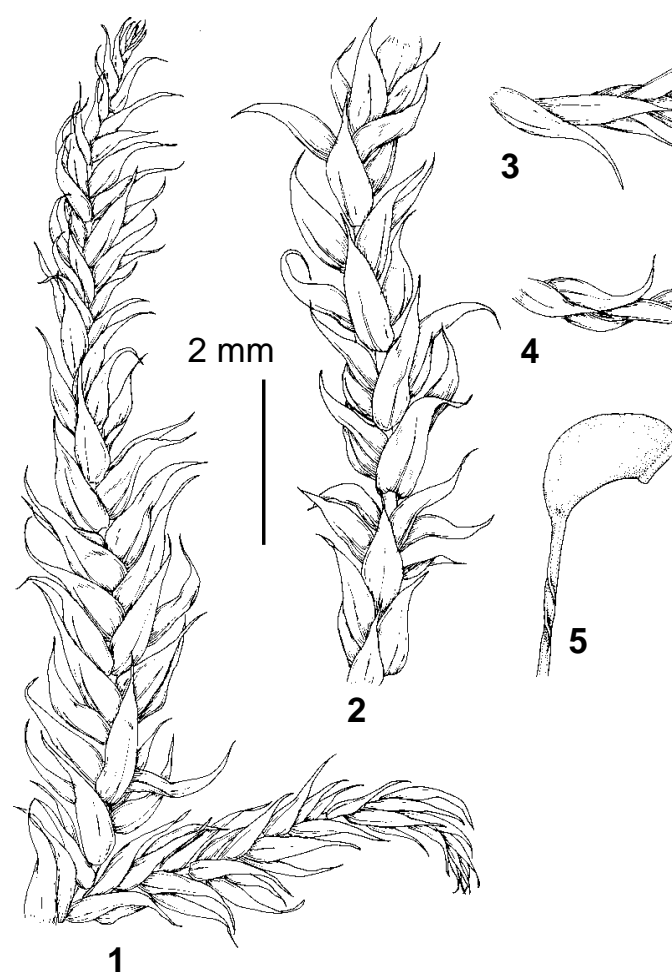

Fig. 3. Sciuro-hypnum oedipodium (Mitt.) Ignatov \& Huttunen (from the lectotype of Hypnum oedipodium Mitt., NY): 1-4 - habit; 5-7 - capsules; 8 - stem leaf; 9 - mid-leaf cells; 10 - basal leaf cells. Scale bars: $2 \mathrm{~mm}$ for $1-7 ; 1 \mathrm{~mm}$ for $8 ; 100 \mu \mathrm{m}$ for $9-10$.

\section{TYPE OF HYPNUM OEDIPODIUM}

Piippo (1983) selected the lectotype of Hypnum oedipodium from several collections of Lyell from Pack River in the Northern Idaho. Two other collections from the same place were designated as syntypes, and are identical, except the collection from Michigan that belongs to S. curtum.

The lectotype is correctly selected, described and illustrated by Piippo (1983). However the collection does not represent plants that are very well developed, thus providing problems with its interpretation. In Fig. 3, 8-10, one of the stem leaves from a small envelope where Piippo has put leaves used for her drawings is illustrated. Fig. 3, 10 shows alar cells typical for plants widespread in the West, and leaf shape (Fig 3,8), mid-leaf cells (Fig. 3, 9) and short capsules (Fig. 3, 5-7), as well as non-complanate foliage (Fig. 3, 1-4). These features demonstrate that the type of Hypnum oedipodium and the species widespread in moun-

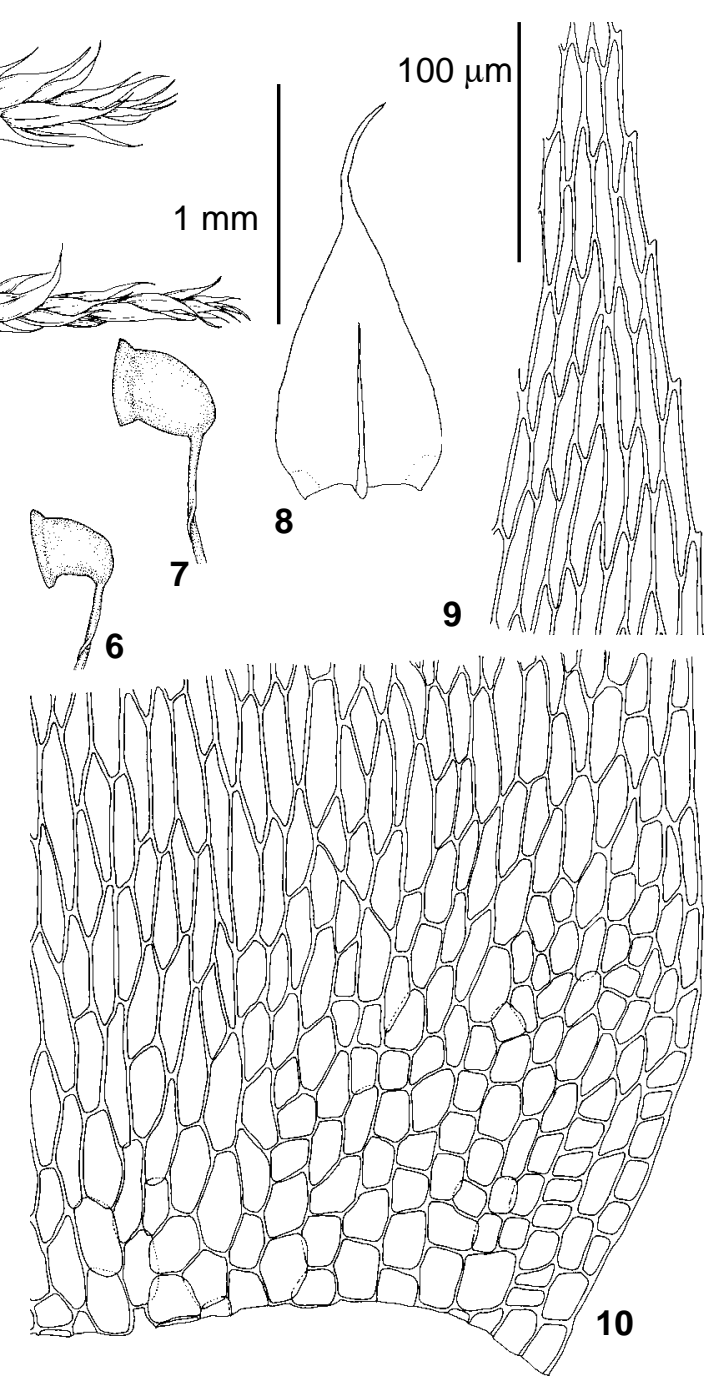

tains of western North America belong to a single species and therefore the name 'oedipodium' should be used for it.

\section{WHAT IS BRACHYTHECIUM HOLZINGERI?}

Grout described this species first as a variety of Brachythecium collinum (Grout, 1901), then raised its status up to species level (Grout, 1922), then decided that it was not a member of Brachytheciaceae at all (Grout, 1928), and finally accepted it again as a good species of Brachythecium (Grout, 1934). This vacillation might look strange to one who has never worked on taxonomy of Brachytheciaceae, where many species exhibit 'intergradations' 'to all other species'. Note that Brachytheciastrum (Brachythecium) collinum is 


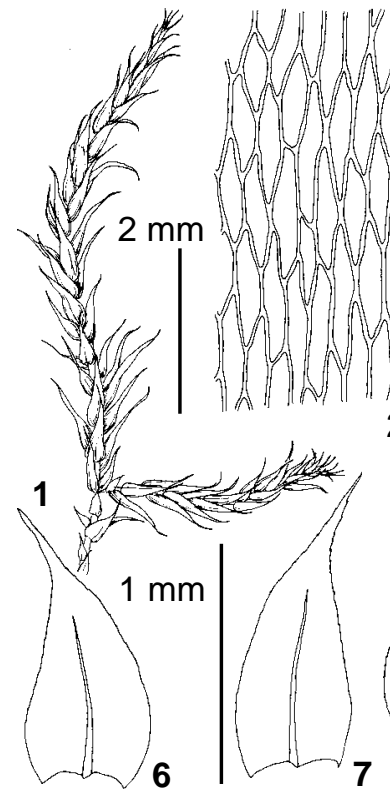

Fig. 4. Sciuro-hypnum oedipodium (Mitt.) Ignatov \& Huttunen (from holotype of Brachythecium collinum var. holzingeri Grout, NY): 1 - habit; 2-3 - mid-leaf cells; 4 - basal leaf cells; 5 - upper leaf cells; 6 -10 - leaves. Scale bars: $2 \mathrm{~mm}$ for $1 ; 1 \mathrm{~mm}$ for $6-10 ; 100 \mu \mathrm{m}$ for $2-5$.

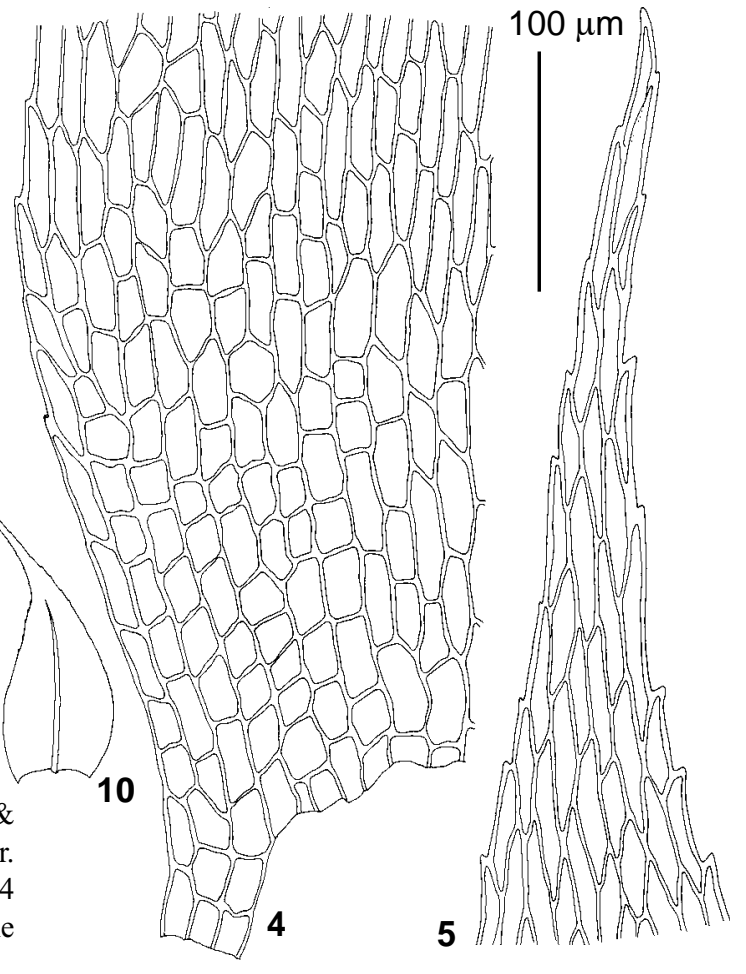

one of the smallest species of Brachythecium s. ampl., while here $B$. collinum var. holzingeri is synonymized with Sciuro-hypnum oedipodium, one of the largest species of Brachythcium s. ampl. This case demonstrates once more the extreme polymorphism of Sciuro-hypnum oedipodium.

Sciuro-hypnum oedipodium (Mitt.) Ignatov \& Huttunen, Arctoa 11: 270. 2002 [2003]. Brachythecium collinum var. holzingeri Grout, Bryologist 4: 236. f. 2a. 1901, syn. nov. Brachythecium holzingeri (Grout) Grout, Bryologist 25: 14. 1922.

The holotype of Brachythecium collinum var. holzingeri in DUKE has a printed label inside the envelope: 'Mosses of North-Western Montana, from the vicinity of lake McDonald, Flathead county, N.-W. Montana. The lake in four miles north of Belton, a station 30 miles east of Kalispell, on the Great Northern Railway. Distributed by J.M. Holzinger. Collected by John M. Holzinger and James B. Blake, July 14-30,

Fig. 5. Sciuro-hypnum oedipodium (Mitt.) Ignatov \& Huttunen (from Colorado, MO): mid-leaf areolation from different leaves of one shoot, showing variation. Scale bar: $100 \mu \mathrm{m}$.
1898, Altitude 3,500-7,000 feet.' Handwritten on this label: 'Base of Sperry glacier, July 25.'

There are three labels glued on the envelope:

1) Rev. N.A.Brachytheciaceae, A.J.Grout, B. collinum var. holzingeri. Leaves larger and more gradually acuminate than in B. collinum. Less serrate leaf, cells broader, [unclear].

2) belong to the collinum group

3) type.

[inside there are also two other labels, one with illegible handwriting, second (printed): 'North American Musci Perfecti issued by A.J.Grout, Ph.D.', pencil handwriting of A.J.Grout 'May 1928, This seems to be Amblystegium Kochii forma'].

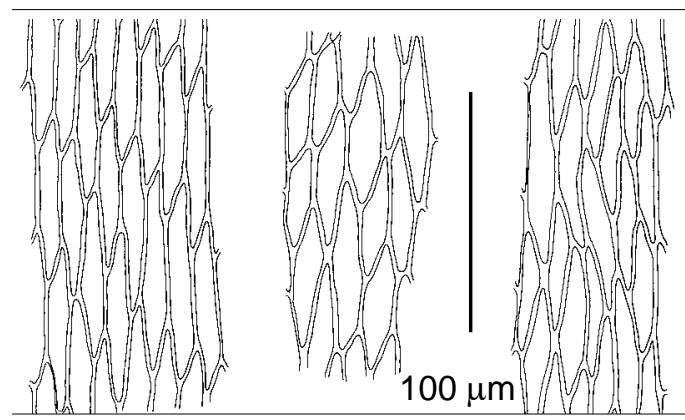


The collection is very poor, comprised mostly of Polytrichum with a few thin shoots of a Brachytheciaceae, which exhibit however the main diagnostic characters of $S$. oedipodium (Fig. 4), and therefore is synonymized with this species.

The type of 'holzingeri' differs from $B$. collinum in the less strong margin serration and less thick-walled cells.

\section{THE OCCURRENCE OF S. OEDIPODIUM IN EURASIA}

The circumspription of $S$. oedipodium in North America has helped in the interpretation of several enigmatic specimens from Russia, that were kept in a folder 'to be described'.

Plants from middle and upper elevations of Caucasus (Fig. 6) could not be comfortably placed in $S$. curtum, because of too long leaves, opaque rather than pellucid alar cells and relatively short capsule. From ITS sequences it appears to be closest to $S$. reflexum, although strikingly different from the latter in the much bigger size of the plants (like large Brachythecium salebrosum or B. rutabulum). The ITS sequence differs from that of $S$. reflexum by just one substitution, but that is the same substitution by which $S$. reflexum differs from the American $S$. oedipodium (see below for details).

In general, $S$. oedipodium seems to be rather rare in Caucasus, and so far we have failed to discover it in other Caucasian countries, although it likely occurs in Georgia. This rarity might also correspond to its rarity in Central European mountains. We found no specimens in MW and LE. At the same time, $S$. oedipodium is likely present there, judging from the overall distribution, and from the descriptions of Limpricht (1896). In addition to Brachythecium curtum, Limpricht also recognized Brachythecium strakei, and the description and illustrations of the latter species are in good agreement with the current concept of $S$. oedipodium. Sciuro-hypnum starkei, as it is understood now (cf. Piippo, 1983, and see also below) does not fit any of Limpricht's descriptions, although according to the key to species 'Brachythecium troemsoense (Kaur. \& Arnell) Limpr.' seems to be fairly close to $S$. starkei, as it has a percurrent costa (however the species description indicates a short costa).

In addition, $S$. oedipodium was found in just one collection from Chukotka, where it could be expected, judging from its distribution in the north-west of North America. The Gil'mimliveem Hot Springs, where it was collected, are not far from another hot springs with the only Asian locality of Plagiothecium undulatum, a species common in Western North America, also exhibiting disjunction to Europe (Afonina, 2004).

THE CURRENTLY KNOWN DISTRIBUTION OF S.

\section{CURTUM AND S. OEDIPODIUM}

The main purpose of this paper is to stress the non-identity of $S$. curtum and $S$. oedipodium that was found during revision of North American and Russian material, thus only a few collections from Eurasia outside Russia were revised and included in the list below. Mostly just one specimen per country is cited, except Russia, U.S.A. and Cana$\mathrm{da}$, where specimens are cited one per state/province/territory/ or other administrative unit.

\section{SELECTED SPECIMENS EXAMINED: Sciuro-hypnum curtum}

EUROPE: RUSSIA : Arkhangelsk Prov.: Kargopol, Churakova \#1238 (MHA); Bashkiria: Malyj Iremel Mt., 700 m, Ignatova \#2/65 (MHA); Belgorod Prov.: Shebekino Distr., Titovka, 12.VII.2006 Maslova (BSU ex MHA); Bryansk Prov.: Zhukovka Distr.: between Zhukovka and Trosna, 23.VII.1974 Makarov (MHA); Chuvashia: Prisursky reserve, V.1999 Moshkovsky (MHA); Kaluga Prov.: Mechivino, 16.VIII.1911 Nikolaevskaya (LE); Karelia: Kindo Cape, 29.VI.1994 Spirina (MW); Komi Republic, 22 km WNW of Syktyvkar, 27.VI.1994 Zheleznova (MHA); Kostroma Prov.: Sharya Distr., Pechenkino, 17.IX.1990 Ignatov (MHA); Kursk Prov.: Centralno-Czernozemny Reserve, 13.VIII.1996 Ignatov (MHA); Leningrad Prov.: Komarovo 26.VII.2007 Kurbatova (LE); Lakhta, 4.IX.1919 anonymous (MW); Mari-El Republic: Bolshaya Kokshaga Reserve, 15.X.1999 Bogdanov et al., (MHA); Moscow Prov.: Taldom Distr., Meldino, 18.VII.1996 Ignatov \& Ignatova (MHA); Murmansk Prov.: Ponoy River, Czernyadjeva 30.VII.1989 (LE); Nizhny Novgorod Prov.: Kerzhensky Reserve, 15.X.1998 Popov \#203 (MHA); Novgorod Prov.: Valdaj Distr., 1.VIII.1981 Morozova (MHA); Perm Prov.: Vishera Reserve, 500 m, 30.VI.1994 Bezgodov \#290 (MW); Pskov Prov.: Mikhailovskoe, 17.VIII.2005 Afonina (LE); Ryazan Province: Oksky Reserve, 2.VI.1968 Bogdanova (MW); Smolensk Prov.: Petrakovo, 16.VII.2004 Ignatov (MHA); Tatarstan: Volzhsko-Kamsky Reserve, 18.VIII.2003 Ignatov \& Ignatova (MHA); Tula Prov.: Yulskie Zaseki, 27.VI.1971 Kurnaev (LE); Udmurtia, Votkinsk Distr., Kostovaty, 17.VII.2000 Munitzyna (MHA); Vladimir Prov.: Gor- 


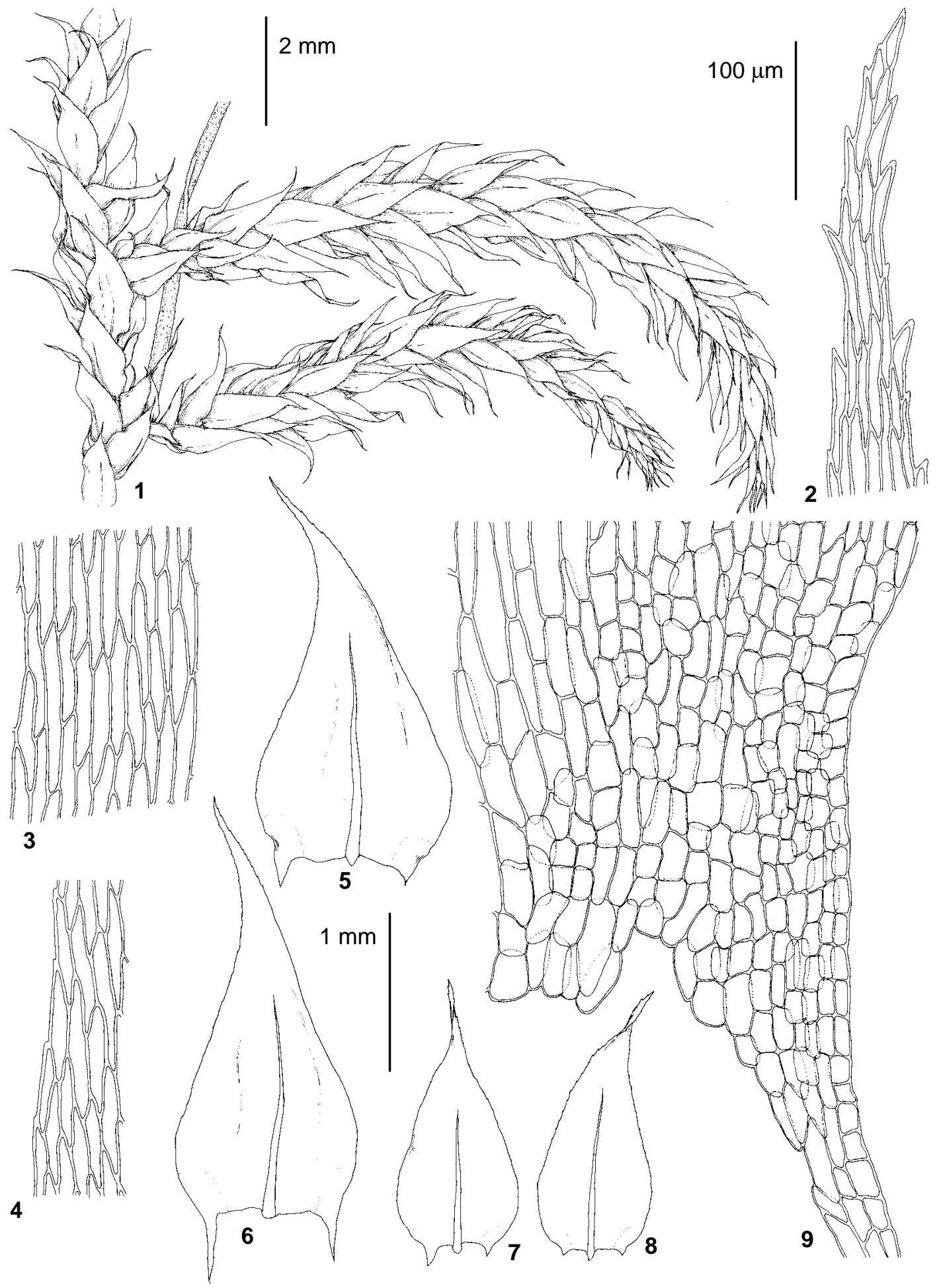

Fig. 6. Sciuro-hypnum oedipodium (Mitt.) Ignatov \& Huttunen (from Russia, Caucasus, Teberda Reserve, 2100 m alt., Ignatov \& Ignatova 06-3038): 1 - habit; 2 - upper leaf cells; 3-4 - mid-leaf cells; 5-6 - stem leaves; 7-8 - branch leaves; 9 - basal leaf cells. Scale bars: $2 \mathrm{~mm}$ for $1 ; 1 \mathrm{~mm}$ for 5-8; $100 \mu \mathrm{m}$ for 2-4, 9 . 
okhovets Distr:: $32 \mathrm{~km}$ NNE of Murom, Seregin \#M1565 (MHA); Vologda Prov.: Sokol Distr., Shorega Creek 22.IX.1990 Ignatov (MHA). GERMANY: Breutel, Flora Germ. Exs. \#387 (LE, sub. Hypnum starkei). POLAND: Ochyra - Musci Pol. Exs. 878,.1182 (MHA, sub. Brachythecium oedipodium). SWEDEN: Stockholm, 25.II.1995 Ignatov (MHA). BELARUS: Grodno Prov., Seregin et al., \#M-1673 (MHA); UKRAINA: Kiev, 19.VII.1986 Virchenko (MHA); ESTONIA: Keeri-Karijдrve Nature Reserve, 31.VIII.2007 Leis (MHA).

ASIA: RUSSIA: Altai Republic: Bolshoye Istyube Creek, 470 m, Ignatov 18/44, 18/9 (MHA); Altaisky Territory: Barnaul, 21.VII.2004 Ignatov (MHA); Amurskaya Prov.: Zeysky Reserve, 5.VIII. 1980 L.I. Abramova (MHA ex IRK); Buryatia: Tompuda, $1500 \mathrm{~m}, 18$.VIII.1956 Bardunov (MHA ex IRK); Evenkiya: Kochumdek Creek, 20.VIII.1991 Shcherbina (MW); Khakassia: Shirisky Distr.: Yarovoe, 12.VIII.1961 Kamenetskaya (MHA ex IRK); Khanty-Mansijsk Autonomous Area: Irtysh, $120 \mathrm{~km}$ downstream from Tobolsk, 8.VIII.1973 Iljina \& Kobeleva (MHA ex IRK); Krasnoyarsk Territory: Boguchansk Distr., Chuny, 4.VIII.1988 Kustova (MHA ex IRK); Kulumys Range, 17.VI.1968 Bardunov (MHA ex IRK); Tomsk Prov.: Kaftannikovo, 11.VIII.1913 (LE); Primorsky Territory: Shkotovo Distr. 15.IX.947 Rosenberg (LE); Tyumen Prov.: Berezovsky Distr. 4.X.1950 (LE); Yakutia: Lensky Distr., Pilka River 25.VII.1999 Ivanova (MHA); Yamalo-Nenetsky Autonomous Area: Salemal, Tenguguy River, 28.VIII.1963 Soldatenkova (MW).

NORTH AMERICA: CANADA: Manitoba, S of Harashville: Mueller-Dombois \#068-13 (MO); New Brunswick: Allen \#2144 (MO); Newfoundland: R.A.C. Waghorne 1.X.[18]93 (MO); Nova Scotia: Schofield \#97254 (MO); Ontario: O.E. \& G.K. Jennings s.n. (MO); Prince Edwards Island: Best, Canadian Musci, \# 380 (MO); Quebeck: Rolland-Germainn \#115 (MO); U.S.A.: Colorado: Grand Co., $2750 \mathrm{~m}$ (9000') spruce-fir forest $6 \mathrm{mi} \mathrm{W}$ of Fraser, 25 Sept 1963 Weber et al. (COLO, B-11091), Connecticut: $26 . X .1979$ J.A. Allen s.n. (MO), Maine: Allen \#14770 (MO); Maryland: Austin herbarium 359 coll. J.D.Smith (NY); Michigan: Cronin 76065 (NY); Montana, Rocky mountains, Columbia Falls: R.S. Williams, North American Mosses, \#259, 19.XI.1896 (NY - two specimes with identical label); New Hampshire: 11.VIII.1878 J.A.Allen (MO); New Jersey: Austin Musci Appalaciani, \#323 (NY); Nov 1866 Austun, (NY); New York: Allen \#27178 (MO); Pennsylvania: Moul 4276 (NY); Vermont: A.J.Grout North American Musci Pleurocarpi, 62 (as Brachythecium starkei); West Virginia: 1.Sept.1922 Bray (NY).

\section{Sciuro-hypnum oedipodium}

EUROPE: RUSSIA: Caucasus: KarachaevoCherkessia: Teberdinskij Reserve, Ignatov \& Ignatova \#05-3850, 05-3038 (MHA).

ASIA: RUSSIA: Chukotka: Gil'mimliveem Hot Springs, 20.VII.1977 A.E.Katenin (LE).

NORTH AMERICA: CANADA: British Columbia: Schofield \#109936 (MO); Yukon: Willians, Noth American Mosses \#718 (NY); U.S.A.: Alaska: Darigo \#2641 (MO); Arizona: Oct 25, 1924 Bartram (NY); Colorado: RM King \& RMGarvey \#B266 (MO); Idaho: Plants of Idaho 1297, coll. Sandberg (MO); Montana: 20.X.1896 R.S. Williams \#346; New Mexico: Buck \#39713 (MO); Utah: Flowers \#2635, 2749, 4785; Washington: Schofield \& Dube \#22352 (MO); Wyoming: Darigo \& Darigo \#3275 (MO).

\section{MOLECULAR DATA}

The protocol of DNA extraction, PCR and sequencing of ITS data was the same as described in Gardiner et al. (2005). Sequences were aligned manually.

Alignment is presented in the Fig. 7.

Results can be commented on as follow:

1. Sciuro-hypnum reflexum, S. starkei, and $S$. oedipodium are very similar in ITS1-2 region, and the former species consistently differ from the two latter species in just one substitution in ITS2 (specific for each of two species).

2. Sciuro-hypnum curtum is different from $S$. reflexum in 8 substitutions ( 3 in ITS1 and 5 in ITS2), and from S. oedipodium and S. starkei in 9 substitutions. Available ITS2 data of Huttunen on S. curtum from Finland, used in the analysis of Huttunen \& Ignatov (2004) and not shown in Fig. 7 has the same 5 characteristic substitutions of this species.

3. Five studied specimens of S. curtum from Europe, East Asia and eastern North America are almost identical, differing in point mutations in individual species, but never corresponding to other specimens of this species, or to other species.

4 . There is only very low variation in the group of species that includes $S$. oedipodium, $S$. reflex$u m$, and S. starkei (and also S. altaicum, S. latifolium, S. glaciale - see Ignatov \& Milyutina, 2007). Only one substitution differentiates $S$. reflexum (some populations) and $S$. starkei, and similarly, only one substitution differentiates $S$. reflexum (some populations) and $S$. oedipodium. 
Fig. 7 (see also next page). Alignment of ITS1 \&2 and gene 5.8S RNA of Sciuro-hypnum species, demonstrating sharp differentiation of $S$. curtum (8 substitutions, marked by squares) from $S$. oedipodium, $S$. reflexum and $S$. starkei, that differs from each other by just one substitution (circles). Specimen data are in Table 1.

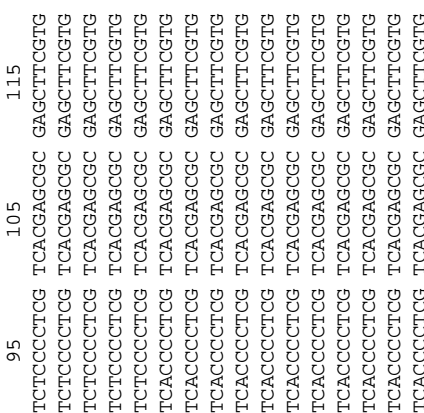

8080808808008000

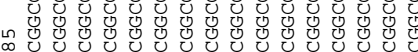

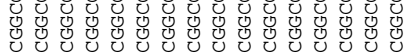

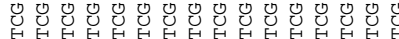

in 8

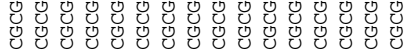

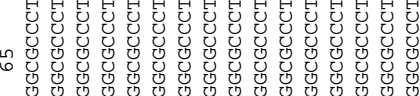

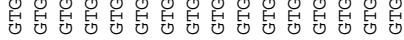

O 0 O 0 O

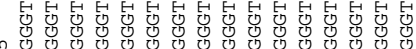

in

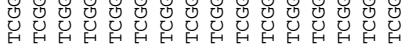

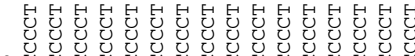

in

TEETEE E E E E E E E

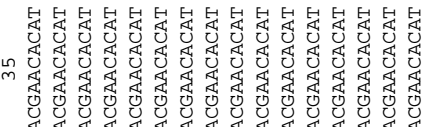

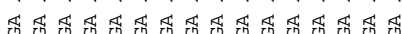

080.8080 .8080

n

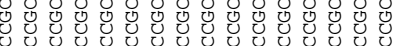

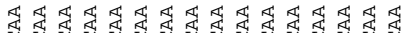

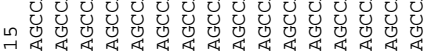

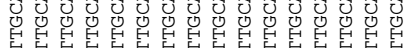

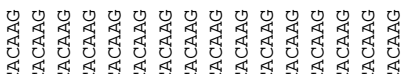

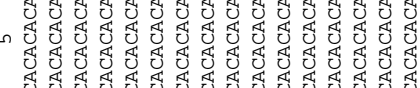

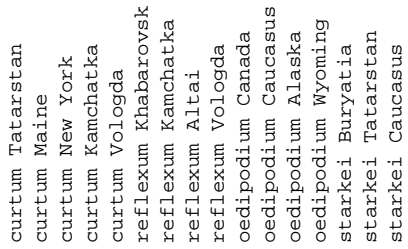

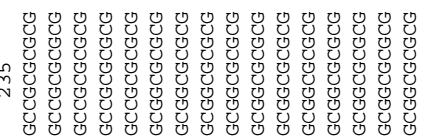

80808080808080

N

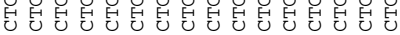

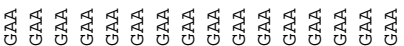

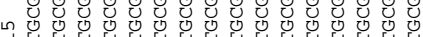

N

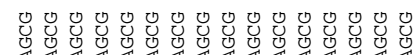

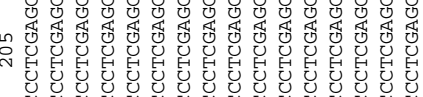

8 8 g g g g g g

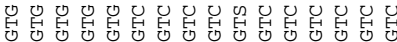

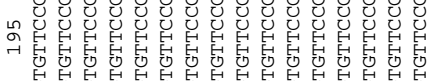

EEEEEEEEEEEEEE

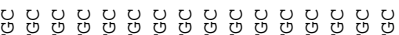

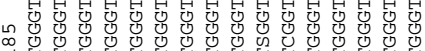

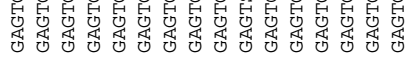

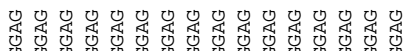

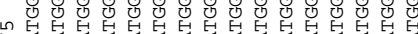

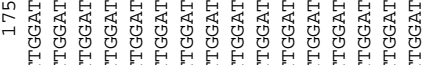

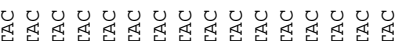

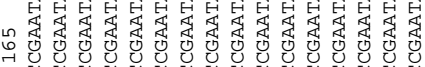

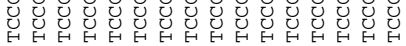

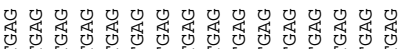

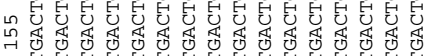

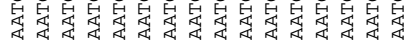

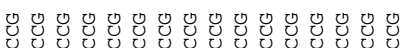

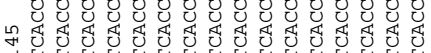

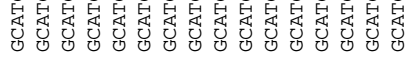

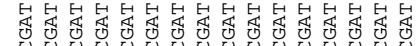

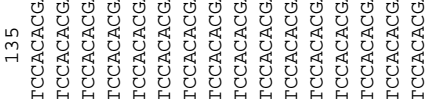

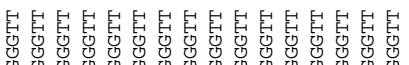

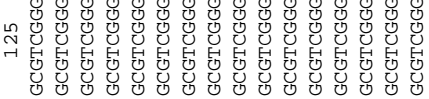

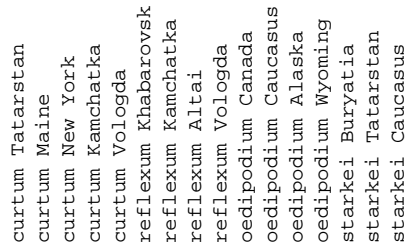

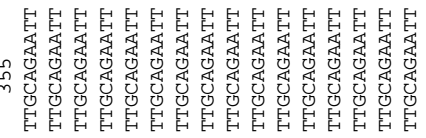

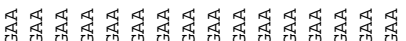

E t t t t t t t t t t t t t t t t t t

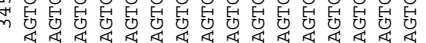

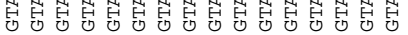

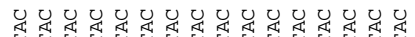

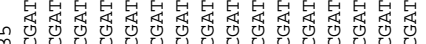

m.

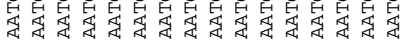

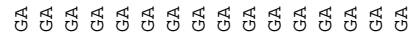

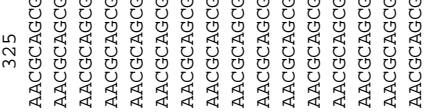

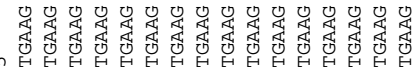

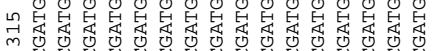

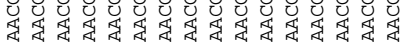

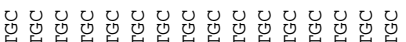

EEEEEEEEEEEEEEEE

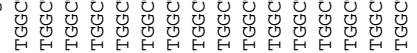

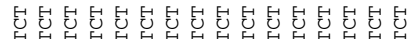

n

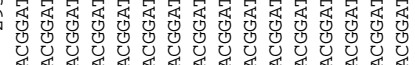

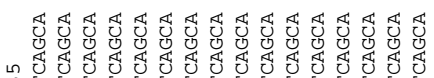

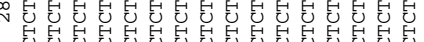

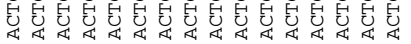

$\delta \delta \delta \delta \delta \delta \delta \delta \delta \delta \delta \delta \delta \delta \delta \delta$

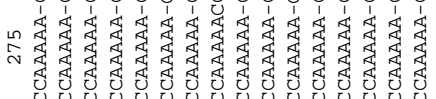

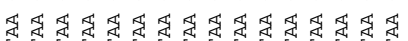

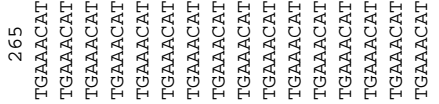

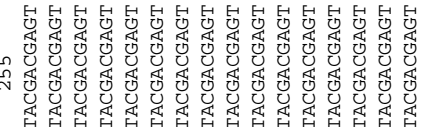

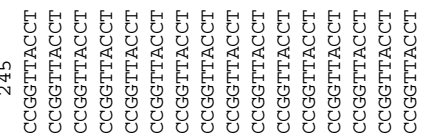

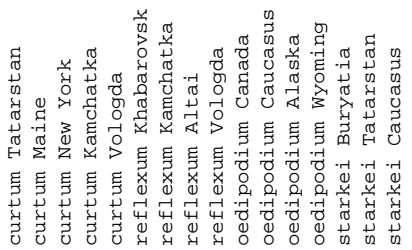


Fig. 7. continued from the previous page.

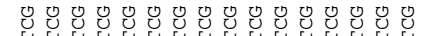

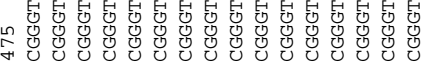

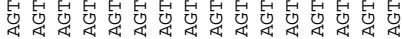
$\forall$
8 10808080 8888888888888888

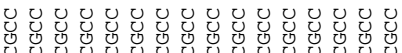

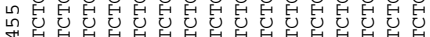

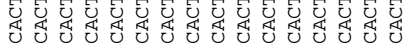
$\cup U U U U U U U U U U U U U U$

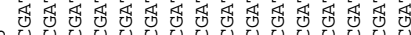

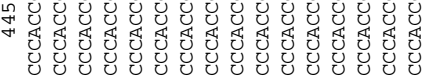
$\left.\begin{array}{l}u \\ \forall\end{array}\right)$

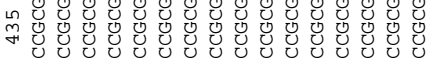

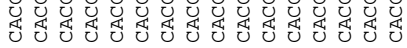
t t t t t t t t t t t t t t t t

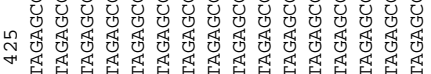

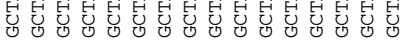

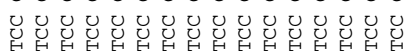

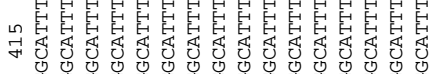

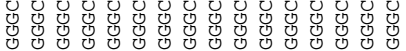

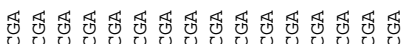

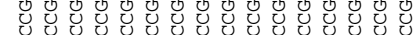

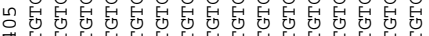

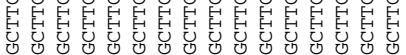

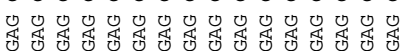

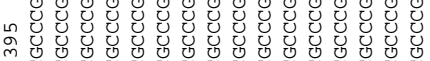

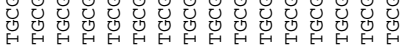

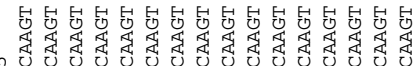

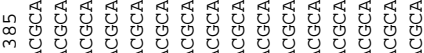

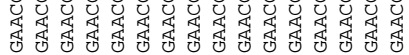

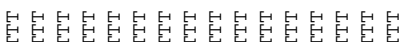

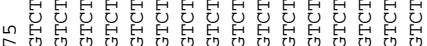

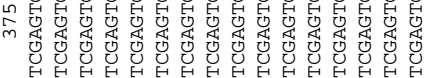

$\delta \delta \delta \delta \delta \delta \delta \delta \delta \delta \delta \delta \delta \delta \delta \delta$

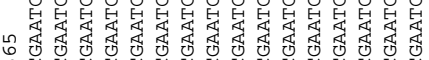

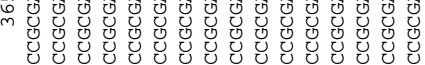

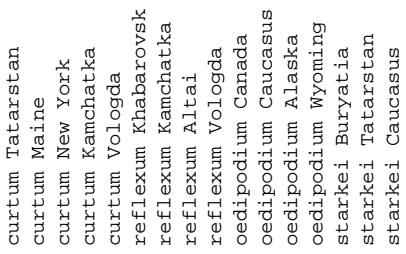

EEEEEEEEEEEEEEEE

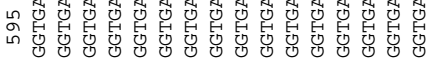

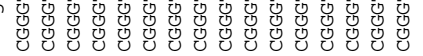

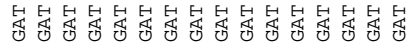

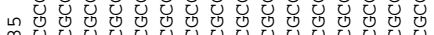

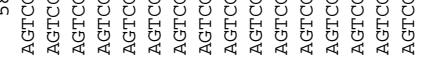

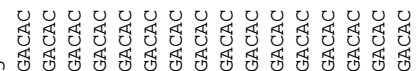

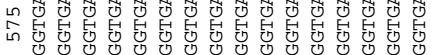
E E E E E E E E E E E 8
8

in 8

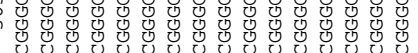

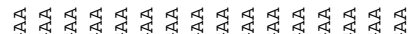

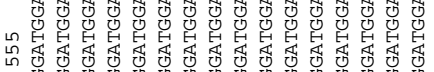
0
0

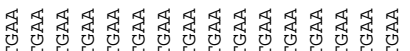

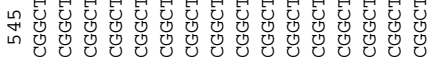
E E E E E E E E E E E E E

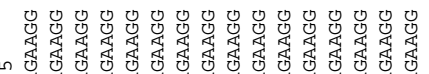

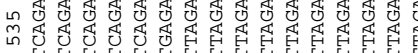

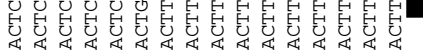

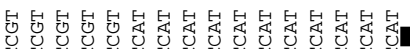

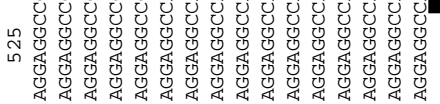

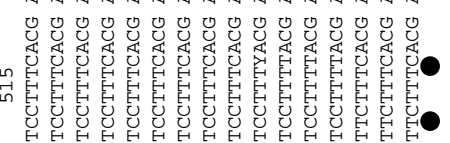

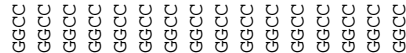

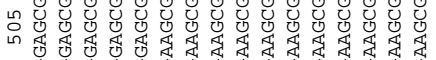
\begin{tabular}{ll}
1 \\
0 \\
\hline
\end{tabular}

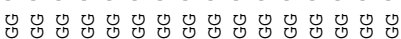

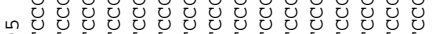

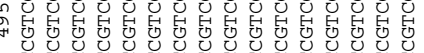
$\forall$
8

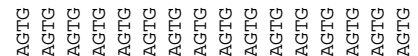
n

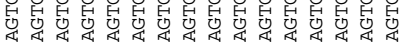

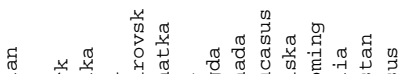

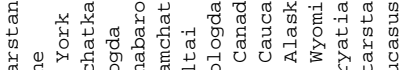

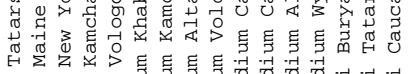

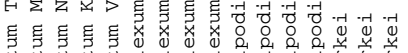

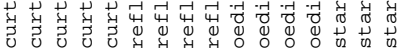

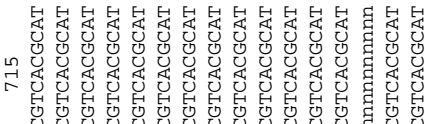

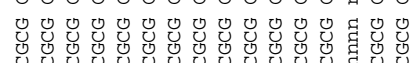

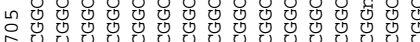

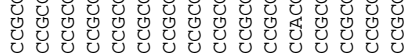

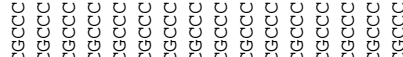

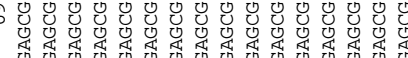
$\left.\begin{array}{l}U \\ \hline\end{array}\right)$

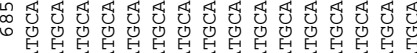

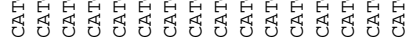

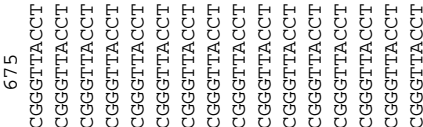

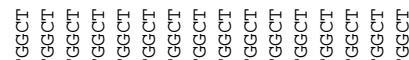
O E E E E E E E E E E E E 88888888888888

苻 可 05050

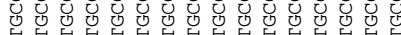
专 \begin{tabular}{c}
0 \\
\hline
\end{tabular}

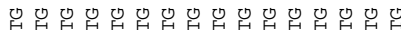
证 0

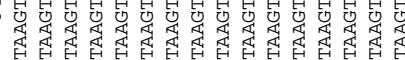

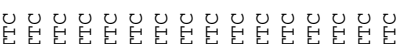
EEEEEEEEEEEEEEEEEEE 8888888888888888

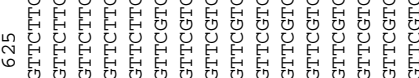
\begin{tabular}{l}
0 \\
\hline \\
\hline
\end{tabular}

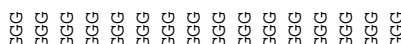

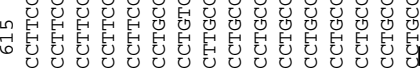

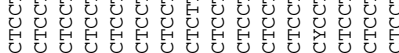

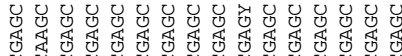
长 G E E E E E E E E E E E E E E

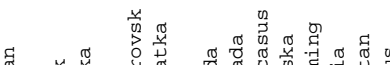

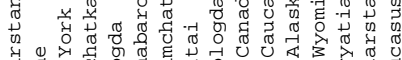

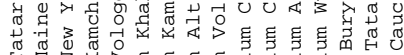

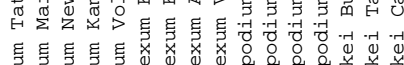

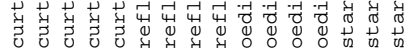




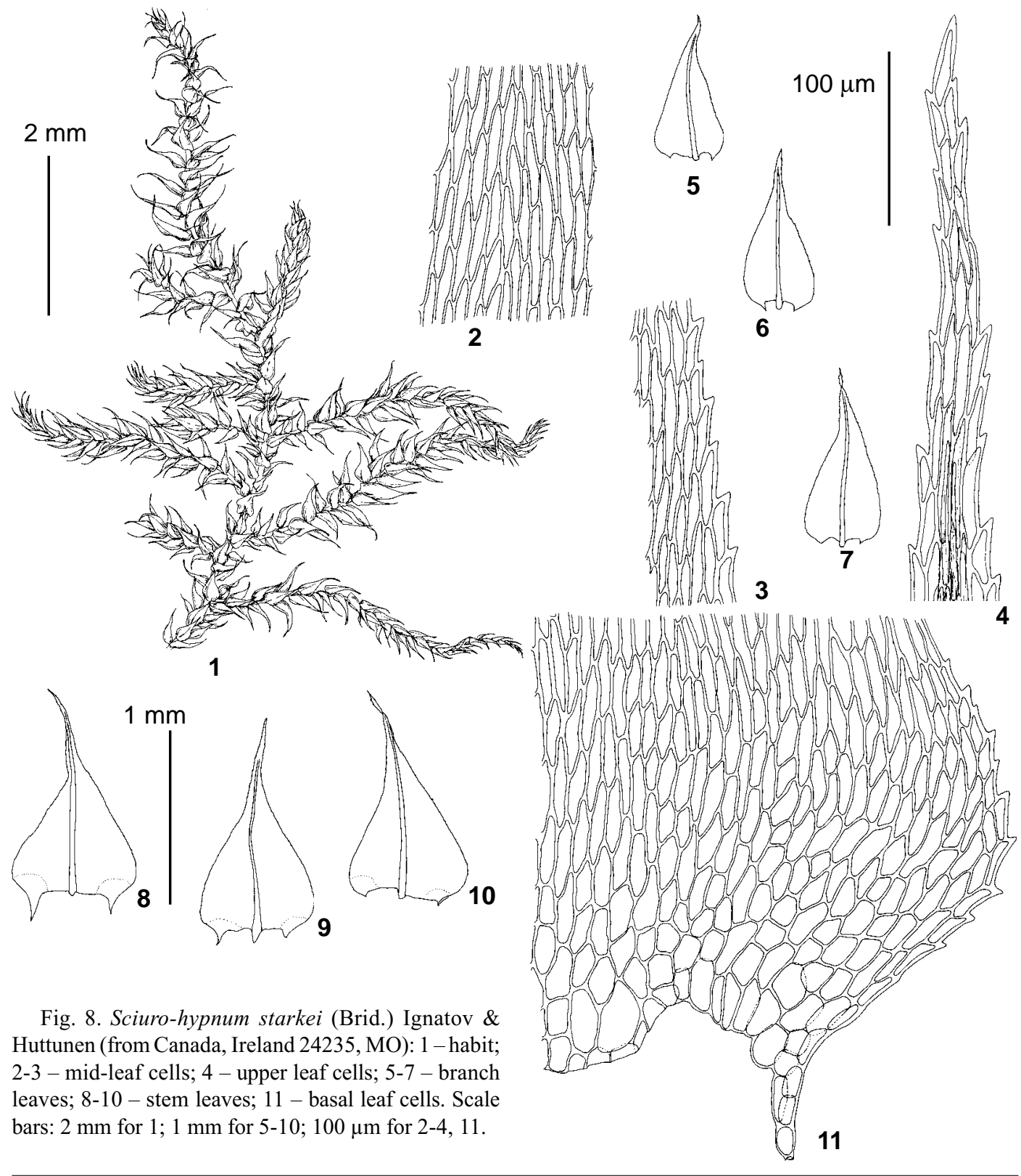

Although the difference is small and includes the only substitution between $S$. reflexum and $S$. starkei, and between $S$. reflexum and $S$. oedipodi$u m$, these differences are consistent with species morphology and constant in the territory of the ca. 4 $000 \mathrm{~km}$ for $S$. starkei, and all around Holarctic for $S$. oedipodium.

\section{WHAT IS SCIURO-HYPNUM STARKEI?}

As most of collections of $S$. oedipodium and S. curtum are still deposited in herbaria (espe- cially in North America) under 'Brachythecium starkei', and this species is considered to be a common one by, e.g. Lawton (1971), a brief comment on $S$. starkei is necessary (for standard description see Ignatov \& Milyutina, 2007).

Sciuro-hypnum starkei is a medium-sized moss that has relatively regular pinnate branching, with rigidly spreading branch leaves and sometimes also stem leaves. Stem leaves are lanceolate-triangular to ovate-triangular, grad- 


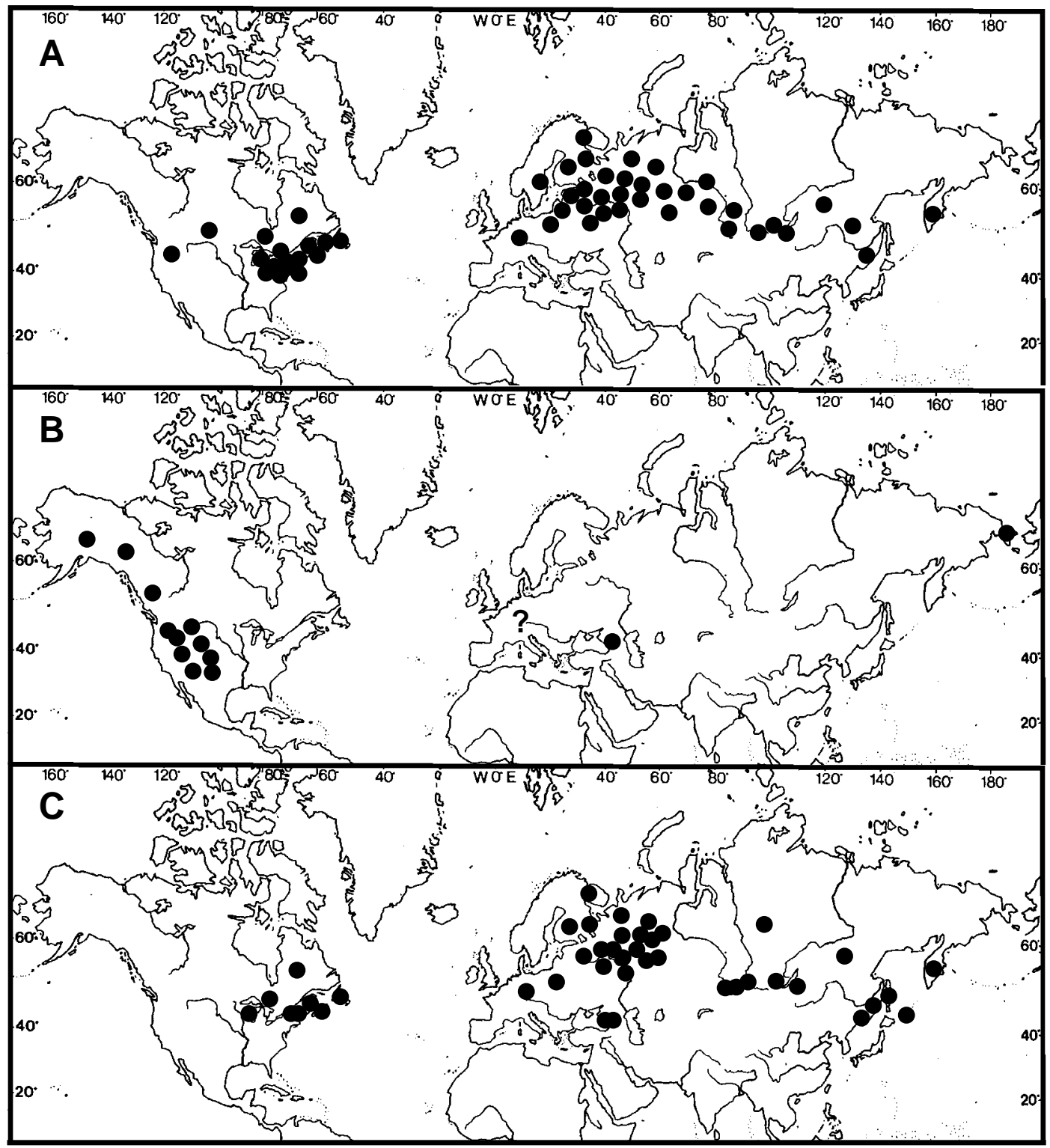

Fig. 9. Distribution of Sciuro-hypnum curtum (A), S. oedipodium (B) and S. starkei (C), basing on revised specimens. Mapping is somewhat schematical, with just one dot per country, except Russia, U.S.A. and Canada, where mapping was up to level of state/province or else administrative units.

ually tapered to apex or sometimes shortly acuminate above, narrowly decurrent. Costa is vanishing in the narrow part of acumen or often percurrent, occasionally ending shortly below acumen. Cells at mid-leaf are 40-60 $\mu \mathrm{m}$, but usually with lengh to width ratio ca. 10:1, so looking very narrow and somewhat flexuose; cells in upper part of branch leaves (occasionally in stem leaves also) are prorate on the dorsal side. Alar cells are enlarged and thinwalled, forming a pellucid group just proximal to the decurrency. Seta is always rough; capsules are relatively short. Under-developed plants differ in more remote and more narrow leaves, retaining however their rigidly spreading appearance.

Rigidly spreading leaves and prorate laminal cells of $S$. starkei result in an apprearance quite 
similar to Kindbergia praelonga; the latter is different however in broader decurrencies, laminal cell proportions 4-8:1 (vs. 8-15:1 in S. starkei), dioicous sexual condition and longly rostrate operculum.

\section{SELECTED SPECIMENS EXAMINED: Sciuro-hypnum starkei}

EUROPE: RUSSIA: Arkhangelsk Prov.: Onega Distr., Ponga, 4.VII.2000 Churakova \#80 (MHA); Bashkiria: Malyj Iremel Mt., Ignatova \#2/111 (MHA); Chelyabinsk Prov., Miass Distr., Novoandreevskaya, 26.VII. 1995 Muldashev \#109 (MW); Chuvashskaya Republic: Prisursky Nature reserve, 12.VIII.2000 Sultanova (MW); Karelia: Olonetsky Distr., Obninsky Forestry Unit, 12.VIII.1984 Volkova (LE); Kirov Prov.: Khalturin Distr., between Aksenovka and Bakulikha (26 km from Nurashi Station) 23.IX.1928 Kolokolnikov (LE); Komi Republic: Pechoro-Ilych Nature Reserve, 27.VI.2000 Bezgodov (Herb. Perm Ped. Univ.); Kostroma Prov.: Manturovo Distr., Khalbuzh 6.VII.1997 Lazareva (MHA); Moscow Prov.: Khimki Distr., Yurlovo 5.VIII.1986 Ignatov (MHA); Murmansk Prov., Polar-Alpine Botanical Garden, $500 \mathrm{~m}$, 31.VII.1998 Ignatov \& Ignatova (MHA); Nizhniy Novgorod Prov.: Kerzhensky Reserve, 100 m, 18.IX.1999 Ignatov (MHA); Novgorod Prov.: Valdai Distr., 25.VII.1981 Vaulina (MHA); Perm Prov.: Vishera State Reserve, 20.VII.1994 Bezgodov (MHA); Sverdlovsk Prov.: Kirovgrad Distr., Visimsky Nature Reserve, 9.VII.2005 Smirnova (MW); Tatarstan, Volzhsko-Kamsky Reserve, Saraly, Ignatov \& Ignatova \#05-2131 (MHA); Tver Prov.: Centralno-Leskoj Reserve, 200 m, 28.VIII.1989 Minaeva (MHA); Udmurtia: Votkinsk Distr., Kostovaty 17.VII.2000 Munitsyna (MHA); Vladimir Prov: Aleksandrovo Distr., Seregin \& Privalova \#M-388 (MW); Vologda Prov.: Sokol Distr., Shorega Creek, 22.IX.1990 Ignatov (MHA); Yaroslavl Prov.: Pleshcheevo Lake, 20.VIII.2001 Ignatov \& Ignatova (MHA). CAUCASUS: Karachaevo-Cherkessia: Teberdinsky Reserve, $2850 \mathrm{~m}$, 8.IX.2005 Ignatov \& Ignatova (MHA); Krasnodar
Territory: Caucasian Nature Reserve, Abago Mt., 1850 m, 20.VII.1960 Artamonova (MW). GEORGIA: South Osetia, Dzava Distr., 13.VIII.1955 Dombrovskaya (MW). GERMANY: Bayrisches Wald, 20.X.1918 Paul (MW). POLAND: Zarnowiec \& al.: Musci Macroregioni Merid. Poloniae Exs. \#302 (MHA).

ASIA: RUSSIA: Altai Territory: Riddera, 25.VIII.1947 Polyakov (MHA); Altai Republic: Kairu-Bazhi Peak, 2050 m, Ignatov \#13/105 (MHA); Amurskaya Territory: Zeya Nature Reserve, 15.VIII.1980 L.Abramova \#126 (MW); Buyatia: Khamar-Daban, Vydrinnaya, 15.VIII.1989 Kazanovsky (MHA ex IRK); Evenkiya: $62^{\circ} 09^{\prime} \mathrm{N}, 91^{\circ} 09^{\prime} \mathrm{E}, 19$.VIII.1992 Szerbina (MW); Irkutsk Prov.: Ulkan, 1.VII.1976 Bardunov (MHA ex IRK); Kamchatskaya Prov.: Kozyrevsk, 220 m, 12.VIII.2004 Czernyadjeva \#64 (LE); Kemerovo Prov.: Tisulsky Distr., Kiya River, 400 m, 7.IX.1971 Vasiliev (MHA ex IRK); Khabarovsk Territory: De Castri Bay, 18.VI.1854 Maximovicz (LE); Primorsky Territory: Lazo Distr., Olkhovaya Mt., 1650 m, 2.X.2006 Ignatov, Ignatova \& Cherdantseva \#062200 (MHA); Sakhalinskaya Prov.: Sakhalin, Dui, V1861 Glehn (LE); Kunashir, Saratovka, Ignatov \#061387 (MHA).

NORTH AMERICA: $\boldsymbol{C A N A D A}$ : New Brunswick: Ireland 13008 (MO); Labrador: R.A.C.Waghorne 1.X.[18]93 (MO); Ontario: Ireland 24235 (MO); Prince Edwards Island: J. Macoun Canadian Mosses \# 293 (MO); Quebeck: Redfearn 37440 (MO); U.S.A.: Maine: Allen 14774, 24555 (MO); Vermont: E.C.Taylor S-697 (NY); Wisconsin: Pursell 6120 (MO).

\section{ACKNOWLEDGEMENTS}

We thank curators of H, IRK, LE, MW, NVS, S, SASY, VLA for making their respective collections available for study; Richard Zander and William Buck for arranging the visit of the first author to MO and NY. We also thank Elena Ignatova for preparing illustrations of species and Angela E. Newon for the English correction. The work was partly supported by the RFBR, grants 07-04-00013 and 06-04-49493.

\section{LITERATURE CITED}

[AFONINA, O.M.] АФОНИНА О.М. 2004a. Конспект флоры мхов Чукотки. - [Moss flora of Chukotka] СПб, БИН РАН [Sankt-Petersburg, Bot. Inst. RAS], 260 pp.

ANDERSON, L. E., H.A. CRUM \& W.R. BUCK 1990. List of mosses of North America north of Mexico. - Bryologist 93: 448-499.

GARDINER, A., M. IGNATOV, S. HUTTUNEN, A. TROITSKY 2005. On resurrection of the families Pseudoleskeaceae Schimp. and Pylaisiaceae Schimp. (Musci, Hyp- nales). - Taxon 54(3): 651-663.

GROUT, A. J. 1901. Two new varieties of Brachythecium. (B.rivulare laxum, B.collinum holzingeri). - Bryologist 4(3): 236 ['48' in N3].

GROUT, A. J. 1922. Brachythecium notes (B. pacificum, B. holzingeri, B. oxycladon forma falcata). - Bryologist 25(1): 13-14.

GROUT, A. J. 1928. Moss flora of North America, north of Mexico. Vol. 3, Pt. 1, Published by the author, Newfane, Vt. \& New-York: 1-62. 
Table 1. Specimen data of the genus Sciuro-hypnum used in analysis, and Genbank accession numbers.

species

curtum Kamchatka

curtum Maine

curtum New York

curtum Tatarstan

curtum Vologda

oedipodium Caucasus

oedipodium Alaska

oedipodium Wyoming

oedipodium British Columbia

reflexum Kamchatka 39

reflexum Khabarovsk

reflexum Vologda

reflexum 'pacificum' Altai

starkei Tatarstan

starkei Caucasus

starkei Buryatiya specimen

Asian Russia, Kamchatka, Czernyadjeva 48-07 (MHA)

GenBank \#

EU715332

U.S.A., Maine, Allen 14770 (MO)

EU715330

U.S.A., New York State, Allen 27178 (MO)

EU715331

European Russia, Tatarstan, Ignatov \& Ignatova 18.VIII.2003 (MHA)

EU715329

European Russia, Vologda, Ignatov \& Ignatova 19-8-2001 (MHA)

EU715333

Russia, Caucasus, Ignatov \& Ignatova 05-3038 (MHA)

U.S.A., Alaska, Darigo 2641 (MO)

U.S.A., Wyoming, Darigo 3275 (MO)

EU715345

EU715346

EU715347

Canada, British Columbia, Schofield 109936 (MO)

Asian Russia, Kamchatka, Chernyadjeva 5.VIII.2002 \#39 (MHA)

EU715344

EU715351

EU715352

European Russia, Vologda Province, Ignatov \& Ignatova 19.VIII.2001 (MHA) EU715353

Asian Russia, Altai, Ignatov 0/442 (MHA)

EU715354

European Russia, Tatarstan, Ignatov \& Ignatova 05-2027 (MHA)

EU715348

Russia, Caucasus, Onipchenko 31-8-1983 \#37-83 (MHA)

EU715350

Asian Russia, Buryatia, Kazanovsky 16.VIII.1989 (MHA)
GROUT, A. J. 1934. Moss flora of North America, north of Mexico. Vol. 3, Pt. 4, Published by the author, Newfane, Vt. \& New-York: 179-277.

HILL, M.O., N. BELL, M.A. BRUGGEMAN-NANNENGA, M. BRUGUÉS, M.J. CANO, J. ENROTH, K.I. FLATBERG, J.-P. FRAHM, M.T. GALLEGO, R. GARILLETI, J. GUERRA, L. HEDENAS, D.T. HOLYOAK, J. HYVÖNEN, M.S. IGNATOV, F. LARA, V. MAZIMPAKA, J. MUÑOZ \& L. SÖDERSTRÖM 2006. An annotated checklist of the mosses of Europe and Macaronesia. - J. Bryol. 28: $198-267$.

HUTTUNEN, S. \& M. S. IGNATOV 2004. Phylogetic analysis of Brachytheciaceae (Bryophyta) based on morphology, and sequence level data. - Cladistics 20: 151-183.

IGNATOV, M. S. 1998. Bryophyte flora of Altai Mountains. VIII. Brachytheciaceae. - Arctoa 7: 85-152.

IGNATOV, M.S., O.M.AFONINA, E.A. IGNATOVA et al. 2006. Check-list of mosses of East Europe and North Asia. - Arctoa 15: 1-130.
IGNATOV, M.S. \& S. HUTTUNEN 2002. Brachytheciaceae (Bryophyta) - a family of sibling genera. - Arctoa 11: 245 296.

IGNATOV, M.S. \& I.A. MILYUTINA 2007. A revision of the genus Sciuro-hypnum (Brachytheciaceae, Bryophyta) in Russia. - Arctoa 16: 73-94.

LAWTON, E. 1971. Moss Flora of the Pacific Northwest. Nichinan, Hattori Bot. Lab., xiii + 362 pp. +195 pl.

LIMPRICHT, K. G. 1896. Die Laubmoose. - In: Rabenhorst, L. Kryptogamen-flora von Duetschland, Oesterreich und der Scheiz. Bd. 3. 1-448.

PIIPPO, S. 1983. On the taxonomy, nomenclature and distribution of Brachythecium starkei (Brachytheciaceae, Musci) and related taxa. - Ann. Bot. Fennici 20: 339349.

REDFEARN, P.L., JR., B.C.TAN \& S. HE 1996. A newly updated and annotated checklist of Chinese mosses $-J$. Hattori Bot. Lab. 79: 163-357. 